\title{
FATORES EMOCIONAIS, QUALIDADE DE VIDA E ADESÃO AO TRATAMENTO EM ADULTOS COM DIABETES TIPO 2
}

\section{EMOTIONAL FACTORS, LIFE QUALITY AND ADHESION OF TREATMENT IN ADULT WITH DIABETES TYPE 2}

\author{
Luciane Ramos ${ }^{1}$ Eleonora Arnaud Pereira Ferreira²
}

\begin{abstract}
RESUMO
Este estudo tem como objetivo verificar a relação entre fatores emocionais (estresse, ansiedade e depressão), qualidade de vida e adesão ao tratamento em adultos com diabetes tipo 2. Participaram 30 pacientes (18 mulheres e 12 homens) entre 33 e 59 anos de idade, matriculados no programa Hiperdia de uma Unidade Básica de Saúde. Os instrumentos utilizados foram os inventários de ansiedade e de depressão de Beck, o inventário de Sintomas de Stress de Lipp (ISSL) e o Questionário geral de saúde e qualidade de vida (SF-36). A adesão ao tratamento foi medida por meio do último resultado do exame de hemoglobina glicada. Os resultados mostraram não haver correlação entre fatores emocionais e adesão ao tratamento. Contudo verificou-se correlação significativa entre tempo de diagnóstico do diabetes e adesão, sugerindo que quanto maior o tempo que o participante apresentava a doença, maior a dificuldade em manter os níveis glicêmicos estabilizados e aderir às orientações prescritas. Observou-se também correlação positiva entre baixa adesão e estrutura familiar. Os resultados sugerem que outros fatores, além dos emocionais, devem ser considerados na análise da adesão ao tratamento.
\end{abstract}

Palavras-chave: diabetes; estresse; ansiedade; depressão; qualidade de vida; adesão ao tratamento.

\begin{abstract}
This study has the purpose of verifying the relationship between emotional factors (stress, anxiety and depression) life quality and adherence to the treatment in adults suffering from type 2 diabetes. Thirty people (18 women and 12 men aging from 33 to 59) participated in the study. All enrolled in the Hiperdia program of a municipal health unit. Instruments used were the inventories of anxiety and Beck's depression, Lipp's inventory of Stress Symptoms The adherence to the treatment was measured by means of the latest result of glycated hemoglobin exam. The results showed that there was no relation between emotional factors and adherence to the treatment. However it was verified a significant correlation between diabetes diagnosis time and adherence, thus suggesting that the longer the time the participant presented the illness, the bigger the difficulty in maintaining the glucose levels stabilized and adhering to the prescribed orientations. It was also observed the positive correlation between low adherence and family structure, predominantly nuclear. The results suggest that other factors besides the emotional ones must be considered in the analysis of adherence to treatment.
\end{abstract}

Key words: diabetes; stress; anxiety; depression; life quality; adherence of treatment.

1 Doutoranda do Programa de Pós-graduação em Teoria e Pesquisa do Comportamento do Núcleo de Teoria e Pesquisa do Comportamento da Universidade Federal do Pará (UFPA). Endereço: Rua Augusto Corrêa, 01, Campus Universitário do Guamá, Belém, Pará. CEP 66.075-110, fone: (91) 81694728,E-mail: lucianeramos@ufpa.br.

2 Professora Doutora do Programa de Pós-graduação em Teoria e Pesquisa do Comportamento do Núcleo de Teoria e Pesquisa do Comportamento da Universidade Federal do Pará (UFPA).

Como citar este artigo: Ramos L, Ferreira EAP. Emotional factors, life quality and adhesion of treatment in adult with diabetes type 2. Journal of Human Growth and Development 2011; 21(3): 867-877.

Artigo submetido em 16.03.11, aceito em 20.08.11. 


\section{INTRODUÇÃO}

O diabetes é uma doença que atinge 220 milhões de pessoas no mundo todo ${ }^{1}$. Segundo a Organização Mundial de Saúde, o diabetes é um problema de saúde pública, causando impacto social e econômico, tanto em termos de produtividade (relacionadas a faltas ao trabalho), quanto de altos custos (relacionados às principais causas de hospitalização). Essas consequências são importantes para que a prevenção e o tratamento do diabetes sejam vistos com mais cuidado pelos serviços de saúde pública.

Estudos epidemiológicos mostram que a prevalência e a incidência do diabetes no Brasil têm aumentado a cada ano. Estima-se que existam 11 milhões de indivíduos vivendo com diabetes, na sua maioria portadores de diabetes tipo 2. Esses dados têm sido preocupantes em razão da inexistência de cura para essa doença e pelas complicações que dela resultam ${ }^{2}$. O diabetes, se não tratado de forma adequada, leva o indivíduo a apresentar, em longo prazo, complicações crônicas e irreversíveis, como disfunção e falência de órgãos, como rins, olhos, nervos, coração e vasos sanguíneos; acarretando doenças, como a neuropatia, a nefropatia, a retinopatia, o infarto do miocárdio, acidentes vasculares e infecções.

O tratamento do diabetes é muito complexo e envolve mudanças no estilo de vida dos pacientes. Cuidados com a automonitoração da glicemia, a prática regular de atividade física, a administração de medicamentos (antidiabéticos orais e/ou insulina) e a adoção de uma alimentação saudável são importantes para manter os níveis glicêmicos estabilizados e, consequentemente, prevenir as complicações crônicas.

A American Diabetes Association ${ }^{3}$ preconiza que variáveis psicológicas e sociais sejam incluídas como parte contínua no manejo da doença. Também destaca a necessidade de que fatores emocionais, como depressão, ansiedade e estresse, sejam avaliados quando há um baixo controle glicêmico, sugerindo que tais fatores são de extrema importância para o seguimento do tratamento e fazem parte dos padrões de cuidados para o diabetes.

\section{Fatores emocionais no manejo do diabetes}

Estudos apontam que pacientes com diagnóstico de doenças crônicas têm maior probabilidade de desenvolver formas patológicas de estresse, ansiedade e depressão4-7. Tais estudos sugerem que esses indivíduos, ao serem expostos a situações aversivas, apresentariam dificuldades no manejo da doença comprometendo a adesão ao tratamento e consequentemente a sua qualidade de vida. No caso específico do diabetes, estudos sugerem haver correlação positiva entre esses fatores e o curso da doença ${ }^{8-11}$.

Com o objetivo de identificar a interferência de fatores emocionais sobre o autocuidado em portadores de diabetes tipos 1 e tipo 2, Santos-Filho e cols. ${ }^{9}$ realizaram três estudos. O Estudo 1, com oito escolares, portadores de diabetes tipo 1; Estudo 2, com 11 portadores de diabetes tipo 2, na faixa etária de 50 a 76 anos, e Estudo 3, com sete participantes de 44 a 66 anos. Nos três estudos, foi utilizado um roteiro de entrevista semiestruturada sobre as reações emocionais dos participantes. As reações emocionais identificadas foram: medo, tristeza, depressão, raiva/revolta e ansiedade/estresse. Essas reações, segundo os autores, estão presentes tanto no momento do diagnóstico como na maneira de lidar com a doença ao longo do tratamento. De acordo com os autores, o diabetes revelou ser uma doença que, independentemente da faixa etária e da etiologia, causa impacto negativo na vida do indivíduo. Dentro dessa concepção, sugerem que os profissionais de saúde precisam estar sensíveis a esses aspectos da vida do paciente, uma vez que as manifestações emocionais podem ter relação com controle glicêmico adequado e com a qualidade de vida do paciente.

Estudo realizado por Maia e Araújo8 ${ }^{8}$ sugere que o perfil psicológico e o grau de aceitação do diabetes tipo 1 pelo paciente podem influenciar diretamente no 
controle glicêmico. Nesse estudo, foi verificada a influência do perfil psicológico dos pacientes, por meio de um questionário que avaliou os sentimentos dos pacientes em relação à doença comparando com seus níveis glicêmicos. Os resultados mostraram que $60,4 \%$ dos pacientes estavam lidando bem ou muito bem com a doença e 13,90\% relataram ter vergonha de falar em público que tinham o diagnóstico de diabetes, ao passo que $60,4 \%$ confirmaram o sentimento de medo em relação a uma crise hipo ou hiperglicêmica em ambiente público. Esses achados são importantes, visto que apontam para uma compreensão mais ampla da doença, sugerindo que aspectos psicológicos são importantes na busca de estratégias de enfrentamento da doença, evitando complicações que o diabetes ocasiona.

Estudo realizado por Silva, Pais-Ribeiro e Cardoso 7 comparou níveis de ansiedade, depressão e estresse entre pacientes com diabetes com e sem complicações crônicas. Os participantes desse estudo foram 316 pacientes com idades entre 16 e 84 anos, sendo que $55,4 \%$ eram do sexo feminino e $59,8 \%$ apresentavam complicações da doença. Os participantes responderam a uma escala de acontecimentos de vida, adaptado para a língua portuguesa por Silva, Pais-Ribeiro, Cardoso e Ramos ${ }^{12}$ e a um inventário de ansiedade e de depressão (Hospital Anxiety and Depression Scale [HADS]). Os dados foram analisados estatisticamente e sugerem que os pacientes com complicações crônicas apresentaram maior nível de depressão que os demais, mas não diferiram quanto à ansiedade. Não houve diferença quanto ao estresse negativo (distresse) entre os participantes, mas os participantes com complicações crônicas mostraram menos estresse positivo (eutresse).

Riveros, Cortazar-Palapa, Alcazar e Sánchez-Sosa ${ }^{13}$ realizaram um estudo cujo objetivo foi avaliar os efeitos de uma intervenção cognitivo-comportamental em pacientes com diabetes e/ou hipertensão. Participaram 51 pacientes de uma unidade de medicina familiar na cidade do México, com média de idade de 54,27 anos, sendo $17 \mathrm{com}$ hipertensão, $27 \mathrm{com}$ diabetes e 7 com diabetes e hipertensão. Foi aplicado o inventário de qualidade de vida e saúde proposto por Riveros, Sánchez-Sosa e Groves ${ }^{14}$, o inventário de ansiedade e o de depressão de Beck (adaptado) e a Escala de enfrentamento de Moos-forma B desenvolvido por Moos, Cronkite, Billings e Finney ${ }^{15}$, além do Sistema de autorregistro diário de adesão e bem-estar, elaborado por Cortazar-Palapa, Riveros e SánchezSosa ${ }^{16}$ que avalia a adesão dos pacientes quanto à medicação, à dieta, à atividade física e aos comportamentos de riscos à saúde. $O$ objetivo foi investigar a qualidade de vida, ansiedade, depressão, estilos de enfrentamento, adesão e bem-estar nesse grupo de pacientes. Os resultados sugerem que as intervenções empregadas promoveram melhora significativa das variáveis investigadas, assim como na diminuição do nível glicêmico e da pressão arterial dos participantes. Com base nesses resultados, os autores concluíram que, no contexto do sistema de saúde, não basta apenas que o paciente receba instruções e informações sobre a doença e o tratamento, sem receber atenção e seguimento cuidadoso por parte dos profissionais de saúde.

Com a proposta de examinar a eficácia de um treino de manejo de estresse em 26 pacientes com diabetes tipo 2, Surwit e cols. ${ }^{10}$ utilizaram o IDATE*, a Escala de estresse percebido e o Questionário geral de saúde SF - 36. Concomitante à aplicação desses instrumentos, foi implementado um programa de educação em diabetes e um programa de manejo de estresse. Os resultados mostraram que o treino de manejo de estresse estava associado a uma pequena, mas significativa redução da hemoglobina glicada (A1C) ao ser comparado com o grupo submeti-

\footnotetext{
* O inventário de Ansiedade Traço-Estado foi adaptado para a língua portuguesa por (Spielberger, Biaggio e Natalício (1979) [17] da versão original de 1970 desenvolvido por Spielberger. Possui uma escala de autorrelato que avalia o estado de ansiedade do indivíduo assim como suas características de personalidade. No Brasil, o Conselho Federal de Psicologia não autoriza o uso deste instrumento.
} 
do somente ao programa de educação em diabetes. Esses achados sugerem a eficácia do treino de controle de estresse no controle glicêmico em pacientes com diabetes tipo 2 .

Em razão da complexidade que envolve o diabetes, a literatura aponta que aspectos relacionados ao próprio paciente, como fatores emocionais e história de vida, são relevantes no atendimento a essa população, uma vez que esses fatores são importantes ao tratamento e prevenção das complicações crônicas que a doença acarreta quando o paciente não se engaja nas orientações fornecidas pelo profissional de saúde. Assim, o objetivo é verificar a relação dos fatores emocionais com a qualidade de vida e a adesão ao tratamento em adultos com diabetes tipo 2 .

\section{MÉTODO}

\section{Participantes}

Participaram do estudo 30 pacientes atendidos no Programa HiperDia de uma Unidade Municipal de Saúde na cidade de Belém-Pará, sendo 18 do sexo feminino e 12 do sexo masculino, com diagnóstico de diabetes tipo 2. A inclusão dos participantes obedeceu aos seguintes critérios: ter entre 25 e 59 anos de idade, estar matriculado no Programa HiperDia há no mínimo seis meses e concordar em assinar o Termo de Consentimento Livre e Esclarecido [TCLE] (Projeto aprovado pelo CEP/NMT, Protosequelas neurológicas (como retinopatia, neuropatia ou amputação), os que estavam fazendo uso de medicamentos prescritos para controle de transtornos psiquiátricos, e aqueles que não concordassem em assinar o TCLE.

\section{Instrumentos}

Os participantes responderam aos seguintes instrumentos:

a) Roteiro de entrevista: roteiro elaborado para este estudo, contendo questões abertas e fechadas, com o objetivo de coletar informações detalhadas acerca das características sociodemográficas e clínicas dos participantes, como idade, sexo, nível de escolaridade, profissão/ ocupação, histórico da doença, assim como a história de adesão ao tratamento do diabetes.

b) Critério de Classificação Econômica Brasil (Associação Brasileira de Empresas de Pesquisa) ${ }^{18}$ : roteiro de entrevista que define a classificação econômica da população brasileira por meio da estimativa do poder de compra dos informantes. Neste estudo, teve como objetivo verificar o nível socioeconômico dos participantes.

c) Inventário de Sintomas de Stress para Adultos (ISSL) ${ }^{19}$ : inventário validado e aprovado pelo Conselho Federal de Psicologia, sendo utilizado em pesquisas e atividades clínicas para o diagnóstico do estresse em adultos. É composto de três quadros referentes aos sintomas (físicos e psicológicos) e às quatro fases do estresse (alerta, resistência, quaseexaustão e exaustão), subdivididos de forma temporal nos sintomas experienciados pelo indivíduo nas últimas 24 horas, na última semana e no último mês.

d) Escalas Beck: Conjunto de quatro inventários utilizados como medida de autoavaliação de depressão, ansiedade, desesperança e tentativa de suicídio, validado para a população brasileira por Cunha $^{20}$. Nesta pesquisa foi utilizado o inventário de ansiedade (BAI) e o de depressão (BDI). O Inventário Beck para Ansiedade (BAI) foi proposto para medir os sintomas comuns de ansiedade. Ele consta de 21 sintomas listados, contendo quatro alternativas em cada um, em ordem crescente do nível de ansiedade. A escala classifica a ansiedade em: mínima (de 0 a 9 pontos); leve (de 10 a 16 pontos); moderada (de 17 a 29 pontos); e grave (de 30 a 63 pontos). O Inventário Beck para Depressão (BDI) compreende 21 categorias de sintomas e atividades, contendo quatro alternativas em cada um, em ordem crescente do nível de depressão. O paciente deve escolher a resposta que melhor se adeque à sua última semana. A soma dos escores identifica o nível de depressão. É proposto o seguinte resultado para o grau de depressão: mínimo (de 0 a 11 pontos); leve 
(de 12 a 19 pontos); moderado (de 20 a 35 pontos); e grave (de 36 a 63 pontos).

e) SF-36 Pesquisa em saúde: Instrumento traduzido e validado para a língua portuguesa de acordo com o International Quality of Life Assessment Project (IQOLA). É um questionário com 36 itens, que engloba oito aspectos (capacidade funcional, aspectos físicos, dor, estado geral da saúde, vitalidade, aspectos sociais, aspectos emocionais e saúde mental). Apresenta um escore final de 0 a 100 , no qual zero corresponde ao pior estado geral de saúde e 100 ao melhor estado.

f) Prontuário multidisciplinar do paciente: documento onde são registrados os atendimentos realizados pelo paciente em suas consultas ambulatoriais, contendo prescrições para o tratamento e resultados de exames laboratoriais, como a hemoglobina glicada.

\section{Ambiente}

Os dados foram coletados nas dependências de uma Unidade Básica de Saúde do município de Belém/Pará. A unidade funciona como um ambulatório para o atendimento em programas de atenção básica em saúde, dentre eles o HiperDia, destinado a pacientes portadores de hipertensão arterial e diabetes mellitus. Nessa unidade, o programa HiperDia é desenvolvido por uma equipe de profissionais formada por médicos, nutricionistas, enfermeiros e técnicos de enfermagem. A coleta dos dados foi realizada em uma sala do ambulatório cedida para as entrevistas com os participantes.

\section{Procedimento}

Foram convidados para participarem da pesquisa os pacientes que estavam na sala de espera na unidade de saúde, aguardando pelo atendimento médico. Aqueles que se enquadravam nos critérios de inclusão e concordaram em assinar o TCLE foram solicitados a responder, individualmente, ao Roteiro de Entrevista, ao Critério de Classificação Econômica Brasil e aos instrumentos: Inventário de Sintomas de Stress de Lipp (ISSL), Inventário de Ansiedade (BAI),
Inventário de Depressão de Beck (BDI) e SF 36-Pesquisa em saúde, nesta ordem. Em seguida, a pesquisadora fez análise do prontuário de cada paciente com a coleta de dados referentes a valores de hemoglobina glicada.

Os resultados foram analisados utilizando-se estatística descritiva e testes de correlação (Qui-Quadrado, Correlação de Pearson, Anova) por meio do programa SPSS versão 15.0, considerando-se as variáveis sociodemográficas, os indicadores de estados emocionais obtidos por meios dos instrumentos padronizados e de adesão ao tratamento obtido por meio do último resultado do exame de hemoglobina glicada. Nesse caso, utilizaram-se os parâmetros adotados pela SBD e pela ADA que sugerem valores iguais ou inferiores a $6,5 \%$ como indicativos de controle do diabetes.

\section{RESULTADOS}

Inicialmente, realizaram-se análises descritivas dos resultados do instrumento e das características sociodemográficas dos participantes. Na tabela 2 descreve-se a comparação entre o sexo dos participantes com valores de hemoglobina glicada acima de $6,5 \%$ quanto ao estresse, depressão e ansiedade. $\mathrm{Na}$ Tabela 3 descrevem-se a frequência e percentual de participantes com valores de hemoglobina glicada acima de 6,5\% que apresentaram comprometimento na qualidade de vida segundo o SF-36 e comparação entre os sexos, finalizando com a tabela 4, em que se comparam a média (M) e desvio padrão (DP) obtidos nos resultados da hemoglobina glicada, estados emocionais (depressão e ansiedade) e aspectos da qualidade de vida de acordo com o sexo dos participantes.

\section{DISCUSSÃO}

$\mathrm{Na}$ área da saúde, alguns estudos vêm realizando importantes avanços na identificação de necessidades associadas a doenças crônicas, como problemas psicofisiológicos, sintomas médicos per- 
Tabela 1: Características sociodemográficas dos participantes $(\mathrm{N}=30)$

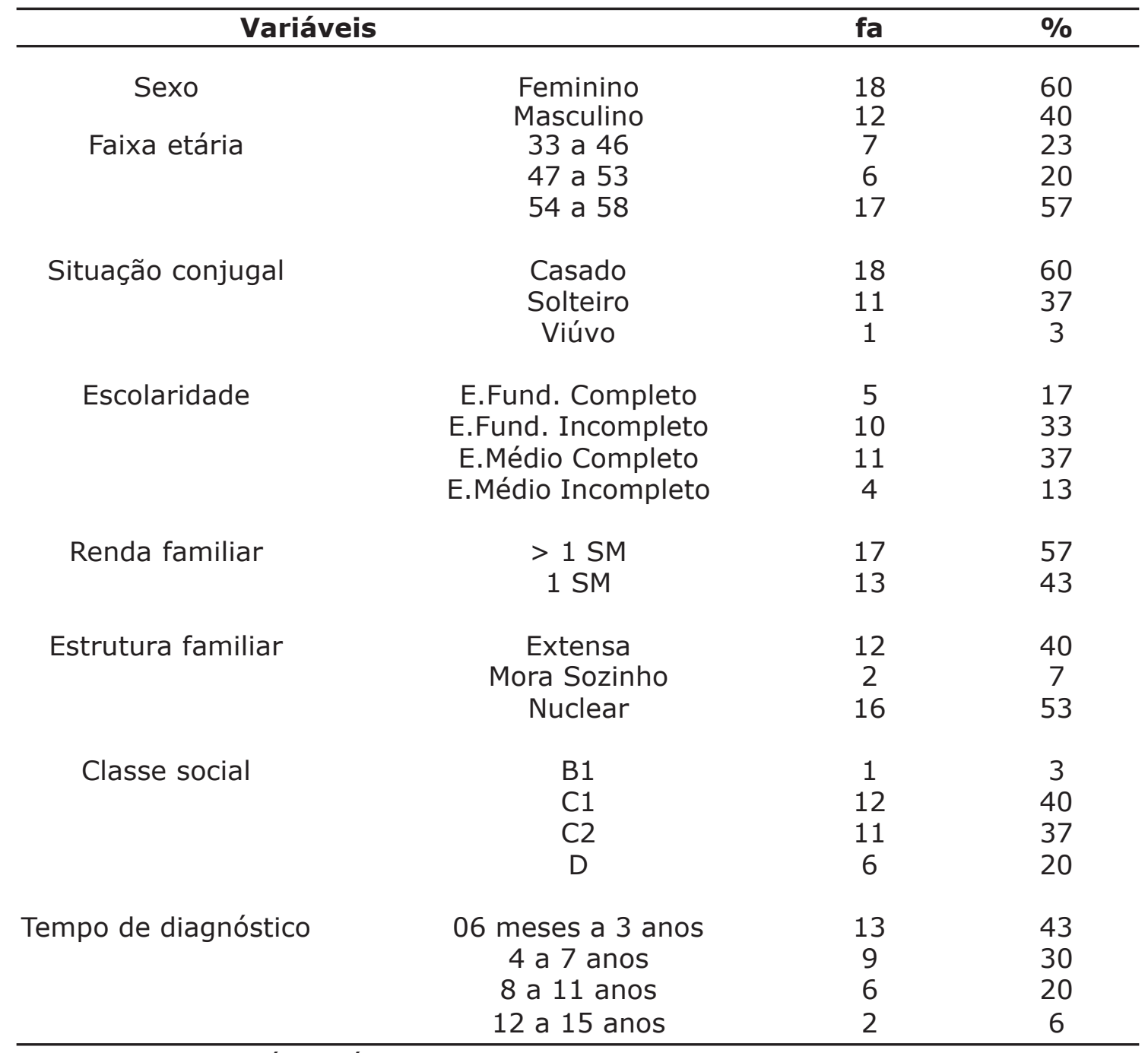

E: Ensino; NS: Salário mínimo

Tabela 2: Comparação entre o sexo dos participantes com valores de hemoglobina glicada acima de $6,5 \%$ quanto ao estresse, depressão e ansiedade $(\mathrm{N}=21)$

\begin{tabular}{lccccc}
\hline \multirow{2}{*}{ Variáveis } & & \multicolumn{2}{c}{ Mulheres (n=14) } & \multicolumn{2}{c}{ Homens $(\mathbf{n = 7})$} \\
& & fa & \% & \% \\
\hline \multirow{2}{*}{ Estresse } & Com estresse & 8 & 57 & 4 & 57 \\
& Sem estresse & 6 & 43 & 3 & 43 \\
Depressão & Mínima & 8 & 57 & 2 & 28,5 \\
& Leve & 4 & 29 & 3 & 43 \\
& Moderada & 1 & 7 & 2 & 28,5 \\
& Grave & 1 & 7 & 0 & 0 \\
Ansiedade & & & & & \\
& Mínima & 11 & 78 & 3 & 43 \\
& Leve & 3 & 22 & 4 & 57 \\
& Moderada & 0 & 0 & 0 & 0 \\
& Grave & 0 & 0 & 0 & 0 \\
\hline
\end{tabular}


Tabela 3: Frequência e percentual de participantes com valores de hemoglobina glicada acima de $6,5 \%(n=21)$ que apresentaram comprometimento na qualidade de vida segundo o SF -36 e comparação entre os sexos

\begin{tabular}{|c|c|c|c|c|c|}
\hline \multirow[b]{3}{*}{ Variáveis } & \multirow{2}{*}{\multicolumn{2}{|c|}{ Feminino $(n=14)$}} & \multicolumn{2}{|c|}{ Sexo } & \multirow{3}{*}{$\begin{array}{c}\text { Coeficiente } \\
\text { de correlação } \\
r\end{array}$} \\
\hline & & & \multicolumn{2}{|c|}{ Masculino $(n=7)$} & \\
\hline & fa & M\% & fa & M\% & \\
\hline Capacidade funcional & 5 & 62,50 & 4 & 48,50 & 0,507 \\
\hline Aspectos físicos & 8 & 25 & 3 & 21,25 & 0,163 \\
\hline Dor & 9 & 47 & 4 & 46,80 & 0,600 \\
\hline Estado geral de saúde & 8 & 47,60 & 7 & 49,75 & 0,277 \\
\hline Vitalidade & 8 & 53,80 & 4 & 47 & 0,697 \\
\hline Aspectos sociais & 6 & 45 & 1 & 36,25 & 0,737 \\
\hline Aspectos emocionais & 5 & 5 & 4 & 27 & 0,772 \\
\hline Saúde mental & 4 & 51,80 & 4 & 54 & 0,340 \\
\hline
\end{tabular}

Nota: Os valores considerados para análise foram os resultados abaixo de $70 \%$ nos escores do teste SF - 36, os quais sugerem comprometimento na qualidade de vida.

Tabela 4: Comparação entre média (M) e desvio padrão (DP) obtidos nos resultados da hemoglobina glicada, estados emocionais (depressão e ansiedade) e aspectos da qualidade de vida de acordo com o sexo dos participantes

\begin{tabular}{|c|c|c|c|c|}
\hline \multirow[b]{3}{*}{ Medidas } & \multicolumn{4}{|c|}{ Sexo } \\
\hline & \multicolumn{2}{|c|}{ Feminino $(n=18)$} & \multicolumn{2}{|c|}{ Masculino $(n=12)$} \\
\hline & M & DP & M & DP \\
\hline Hemoglobina glicada & 8,18 & 2,35 & 6,49 & 1,68 \\
\hline Depressão & 13,88 & 11,72 & 15,08 & 7,98 \\
\hline Ansiedade & 7,94 & 5,02 & 11,16 & 8,13 \\
\hline Capacidade funcional & 82,38 & 14,40 & 72,66 & 23,72 \\
\hline Aspectos físicos & 41,12 & 42,21 & 46,67 & 40,35 \\
\hline Dor & 68,57 & 26,88 & 65,14 & 26,07 \\
\hline Estado geral de saúde & 60,52 & 15,34 & 54,67 & 22,87 \\
\hline Vitalidade & 69,35 & 19,54 & 60,46 & 23,48 \\
\hline Aspectos sociais & 77,91 & 22,27 & 76,87 & 22,69 \\
\hline Aspecto emocional & 48,51 & 43,49 & 43,89 & 48,11 \\
\hline Saúde mental & 79,24 & 20,25 & 62,14 & 25,23 \\
\hline
\end{tabular}

sistentes, sendo desenvolvidas avaliações válidas para medir resultados clínicos em várias culturas, destacando a importância do sexo, da idade, da diversidade racial e étnica, a partir das mais variadas intervenções.

A doença crônica é incurável e de origem não contagiosa, caracterizada por um longo período de latência, curso prolongado, provocando incapacitação e com alguns fatores de risco bem conhecidos, tal qual se caracteriza diabetes tipo 2 . Como a maioria das doenças crônicas é associada ou causada por uma combinação de fatores sociais, culturais, ambientais e comportamentais. Dessa maneira, objetivou-se avaliar se fatores emocionais como estresse, ansiedade e depressão têm relação com a qualidade de vida e a adesão ao tratamento em adultos com diabetes tipo 2.

A Tabela 1 apresenta as características sociodemográficas dos participantes. Observa-se que $60 \%$ da amostra eram do sexo feminino e $40 \%$ do sexo masculino. A maioria estava na faixa etá- 
ria de 54 a 58 anos (57\%), era casada (60\%), possuía nível de escolaridade correspondendo ao Ensino Médio completo $(37 \%)$, era pertencente à classe social C1 (40\%), vivendo com mais de um salário mínimo (57\%) em família predominantemente nuclear (53\%).

A análise dos valores de hemoglobina glicada registrados nos prontuários indicou que $70 \%$ dos participantes estavam com valores acima de $6,5 \%$, indicando dificuldade no controle do diabetes. Destes, $67 \%$ eram do sexo feminino $(n=14)$ e $33 \%$ do sexo masculino $(n=7)$. Os valores de hemoglobina glicada desses participantes variaram de 6,8 a $14 \%$ e o tempo de diagnóstico do diabetes foi de seis meses a 15 anos.

Em seguida, por meio do teste estatístico Qui-Quadrado $\left(c^{2}\right)$ fez-se análise da correlação entre adesão ao

tratamento e as variáveis sociodemográficas sexo, faixa etária, estado civil, escolaridade e renda familiar. Nessa análise foram considerados somente os 21 participantes (14 mulheres e sete homens) que apresentaram resultados de hemoglobina glicada sugerindo dificuldade no controle do diabetes. Os resultados obtidos indicaram que não houve uma relação estatisticamente significante entre essas variáveis.

Foi analisada também a relação entre adesão ao tratamento e a estrutura familiar dos participantes. Os resultados mostraram haver uma associação significante entre essas variáveis, isto é, aqueles participantes que apresentaram baixa adesão ao tratamento eram aqueles que possuíam estrutura familiar predominantemente nuclear $(r=0,047)$.

Os resultados obtidos por meio dos instrumentos que avaliaram os fatores emocionais foram analisados primeiramente de acordo com o sexo dos participantes com dificuldade de adesão. Quanto ao estresse, observou-se que não houve diferença significativa entre os sexos, pois $57 \%$ das mulheres e $57 \%$ dos homens obtiverem resultados no ISSL sugerindo presença de estresse, conforme apresentado na Tabela 2.

Quanto ao inventário de depressão, as participantes do sexo feminino apresentaram predominantemente percen- tuais sugestivos de depressão mínima $(57 \%)$ e leve (29\%). Os participantes do sexo masculino apresentaram escores mais elevados, com resultados indicando depressão leve (43\%) ou moderada $(43 \%)$.

No inventário de ansiedade, a maioria das mulheres obteve resultado indicando presença de ansiedade mínima $(78 \%)$, enquanto a maioria dos homens $(57 \%)$ apresentou ansiedade leve.

Com base nesses dados, pode-se inferir que nesta população não houve diferença significativa entre os sexos com relação aos níveis de estresse, ansiedade e depressão. Apesar dos resultados apresentarem percentuais diferentes, esses dados apontam que não houve diferença estatisticamente significativa entre os sexos, ou seja, nesta população os fatores emocionais não diferiram em relação aos sexos.

Para a análise das correlações entre qualidade de vida, avaliada pelo SF36 (incluindo Capacidade funcional, Aspectos físicos, Dor, Estado geral de saúde, Vitalidade, Aspectos sociais, Aspectos emocionais e Saúde mental), e a dificuldade de adesão ao tratamento $(n=21)$, utilizou-se a correlação de Pearson com nível de significância de $p>0,05$. A Tabela 3 mostra o resultado desta análise.

Pelos resultados obtidos, houve algumas diferenças entre os sexos. Os participantes do sexo masculino tiveram baixos escores na capacidade funcional $(48,5 \%)$, vitalidade $(47 \%)$ e aspectos sociais $(36,25 \%)$, comparados aos escores dos participantes do sexo feminino. No que se refere aos aspectos emocionais, os participantes do sexo feminino apresentaram escores de aspectos emocionais muito baixos $(5 \%)$. Observa-se que esses dados, de um modo geral, indicam um nível de comprometimento nesses aspectos da qualidade de vida dos participantes. Entretanto os testes de correlações estatísticas indicaram que a baixa adesão não foi significativa para a qualidade de vida dos participantes

Fez-se a análise da relação entre o tempo de diagnóstico de diabetes dos participantes e a adesão ao tratamento, por meio do teste de correlação de 
Pearson com nível de significância de $p=0,01$. Obteve-se resultado igual a $r=$ 0,591 . Com base nesse resultado, podese supor que existe correlação positiva de grau moderado entre o tempo de diagnóstico e a adesão, ou seja, quanto maior o tempo de diagnóstico do diabetes apresentado pelo participante, maior o nível de hemoglobina glicada, indicando baixo índice de adesão ao tratamento.

Utilizou-se a Anova com a finalidade de comparar as médias e desvios-padrão dos participantes quanto à qualidade de vida, fatores emocionais e adesão ao tratamento. A Tabela 4 apresenta esses resultados.

Com relação aos aspectos da qualidade de vida dos participantes, houve diferença significativa no que diz respeito à capacidade funcional, estado geral de saúde e saúde mental, pois os participantes do sexo masculino demonstraram estar mais comprometidos nesses aspectos da qualidade de vida que os participantes do sexo feminino.

Com relação aos fatores emocionais (depressão e ansiedade) não houve diferença significante nas médias encontradas entre mulheres e homens.

Finalmente, quando analisada a presença de fatores emocionais relacionados à qualidade de vida e aos valores de hemoglobina glicada, os resultados revelaram que, ainda que não se verificassem diferenças estatisticamente significantes em relação à adesão, estas estão presentes.

Em geral os resultados mostraram que nesta população não houve uma associação estatisticamente significante entre fatores emocionais e adesão ao tratamento (avaliado por meio da hemoglobina glicada), o que indica que os fatores emocionais não foram variáveis determinantes para a falta de adesão. Entretanto, os dados indicam que mesmo não havendo relação causal entre essas variáveis, destaca-se a presença de alterações em fatores emocionais nos indivíduos que convivem com diabetes ${ }^{4,5,6,7}$.
Outras variáveis correlacionadas, como o maior tempo de diagnóstico e os maiores valores de hemoglobina glicada, foram variáveis significativas para a indicação da baixa adesão dos participantes. Contudo, a baixa adesão não foi estatisticamente significante para a qualidade de vida dos participantes.

De um modo geral, este estudo demonstrou não existir diferenças estatisticamente significativas entre os participantes com relação às características sociodemográficas, como sexo, estado civil, quanto à adesão ao tratamento, nem uma correlação estatisticamente significativa entre esta e a idade e escolaridade desses.

Apesar de tais achados, não se pode descartar a presença de associação entre essas variáveis, pois a literatura corrobora que estas são de extrema importância para o desenvolvimento de estilos de vida adequados para que o paciente conviva com a doença de forma mais saudável ${ }^{21}$.

Outro achado muito relevante verificado neste estudo foi o nível de adesão correlacionado com a estrutura familiar nuclear dos participantes, esse dado indica que os participantes com maior nível de hemoglobina glicada tinham estrutura predominantemente nuclear, o que corrobora alguns estudos em que a falta de apoio da família é apontada como um fator que dificulta a adesão de uma doença crônica, neste contexto o diabetes 22 .

Esses dados sugerem que outras variáveis podem estar interferindo na adesão desses participantes. Contudo, neste estudo, não foi possível identificar quais fatores contribuíram para a baixa adesão, sugerindo a necessidade de estudos longitudinais acompanhando o indivíduo no processo de adesão ao tratamento.

É necessário considerar a importância dos fatores emocionais em indivíduos que convivem com doenças crônicas como o diabetes, oferecendo suporte emocional e treino em estratégias de enfrentamento da doença. 


\section{REFERÊNCIAS}

1. World Health Organization. Diabetes, Fact sheet, [periódico online]. 2011 Jan [acesso em 3 mar 2011]. Disponível em http:// www.who.int/ mediacentre/factsheets/fs312/en/.

2. Sociedade Brasileira de Diabetes SBD. Cuidados de Enfermagem em diabetes mellitus. Em Manual de Enfermagem da Sociedade Brasileira de Diabetes. Citado em 25/03/2011, Disponível em: http://www.diabetes.org.br/attachments / 1118_1324_manual_enfermagem.pdf.

3. American Diabetes Association. Standards of medical care in diabetes. Diabetes Care, 34 (1, Supplement). 2011 [acesso em 25 mar 2011 Disponível em: http:// care.diabetesjournals.org/content/ 34/Supplement_1/S11.extract.

4. Ataíde M.B. C., \& Damasceno M. M. C. Fatores que interferem na adesão ao autocuidado em diabetes. 2006Revista de Enfermagem UERJ, RJ, 14 (4): 518-23. Retirado em 09/ 07/2008, de Disponível em: http:// www.portalbvsenf.eerp.usp.br/ scielo.php?script $=$ sci_pdf\&pid $=$ S0104$35522006000400005 \&$ Ing $=$ pt\&nrm $=$ iso\&tlng $=$.

5. Carvalho, N. S., Ribeiro, P. R. Ribeiro, M. Nunes, M. P. T. Cukier, A. \& Stelmach R. Asma e doença pulmonar obstrutiva crônica: Uma comparação entre variáveis de ansiedade e depressão, Jornal Brasileiro de Pneumologia, 2007; 33(1), 1-6.

6. Chaves, E. C. \& Cade, N. V. Efeitos da ansiedade sobre a pressão arterial em mulheres com hipertensão $R e$ vista Latino-americana de Enfermagem março-abril; 2004; 12(2), 162-167.

7. Silva, I., Pais-Ribeiro J., \& Cardoso H.. Dificuldades em perceber o lado positivo da vida? Stress em doentes diabéticos com e sem complicações crónicas da doença. Avaliação Psicológica, 3(22), 597-605. 2004. Retirado em 27/11/2007, de Disponível em: www.scielo.oces.mctes.pt/pdf/ aps/v22n3/v22n3a14.pdf.

8. Maia, F. F. R. \& Araújo L. R.. Aspectos psicológicos e controle glicêmico de um grupo de pacientes com diabetes mellitus tipo 1 em Minas Gerais. Arquivos Brasileiros Endocrinologia e Metabologia, 2004; Vol. 48, n 2, p 261-266.

9. Santos-Filho, C. V., Rodrigues, W. H. C. \& Santos R. B. Papéis de auto-cuidado - Subsídios para enfermagem diante das reações emocionais dos portadores de diabetes mellitus. Escola Anna Nery - Revista de Enfermagem, 2008, 12(1), 125-129.

10. Surwit RS, van Tilburg MA, Zucker $\mathrm{N}$, McCaskill CC, Parekh $\mathrm{P}$, Feinglos MN, Edwards CL, Williams P, Lane JD. Stress management improves longterm glycemic control in type 2 diabetes. Diabetes Care, 2002, 25(1):30-4.

11. Thomas, J., Jones, G., Scarinci, I. \& Brantley, P.. A descriptive and comparative study of the prevalence of depressive and anxiety disorders in low-income adults with type 2 diabetes and other chronic illnesses. Diabetes Care, 2003, 26, Number 8.

12. Silva, I., Pais-Ribeiro, J., Cardoso, H., \& Ramos, H. Contributo para a adaptação da Life Events Survey (LES) à população diabética portuguesa. $R e$ vista Portuguesa de Saúde Pública, 2003, 2, 49-60.

13. Riveros, A., Cortazar-Palapa, J., Alcazar, F. L. \& Sánchez-Sosa, J. J. Efectos de una intervención cognitivo-conductual en la calidad de vida, ansiedad, depression y 
condición médica de pacientes diabeticos e hipertensos esenciales. International journal of clinical and health psychology, septiembre, año/ vol. 5 numero 003, Asociación Española de Psicología Conductual (AEPC), granada, Espanha, 2004, p, $445-462$.

14. Riveros, A., Sánchez-Sosa, J. J. e Groves, M. Inventario de calidad de vida y salud, InCaVisa: México Universidad Nacional Autonóma de México; 2004.

15. Moos, R., Cronkite, R., Billings, A. e Finney, J. Health and daily living form manual. Social Ecologic Laboratory, Department of Psychiatry and Behavioral Science. Palo Alto, CA: Veterans Administration and Standford University medical Center; 1986.

16. Cortazar-Palapa, J., Riveros, A., Sánchez-Sosa, J. J. Herramientas para la toma de decisiones en terapia cognitivo-conductual en escenarios médicos: el uso del sistema de autorresgistro. Ponencia presentada en el XII Congreso Mexi- cano de Psicología, Guanajuato, México; 2004.

17. Spielberger, C.D.; Biaggio, A.; Natalicio, L.F. - Manual do Idate- Rio de Janeiro, R.J., CEPA, 1979

18. Associação Brasileira de Empresas de Pesquisa Critério de Classificação Econômica Brasil. Retirado em: 03/ 01/2010. Disponível em http:// www.abep.org/novo/Content. aspx? ContentID =302.

19. Lipp, M. E. N. Inventário de sintomas de stress para adultos de Lipp. São Paulo: Casa do Psicólogo; 2000.

20. Cunha, J. A.. Escalas Beck. São Paulo: Casa do Psicólogo; 2001.

21. Silva, I. Pais-Ribeiro, J. Cardoso, H. Adesão ao tratamento da diabetes Mellitus: A importância das características demográficas e clínicas. Revista Referência II a Série, 2006; nº 2.

22. Lopes, M. C. L., Marcon, S. S. A hipertensão arterial e a família: a necessidade do cuidado familiar Revista Esc de Enfermagem USP, 2009; 43(2): 343-50. 